\title{
Hot ISM in young elliptical galaxies
}

\author{
Dong-Woo Kim ${ }^{1}$ \\ ${ }^{1}$ Smithsonian Astrophysical Observatory, 60 Garden Street, Cambridge, MA 02138, USA \\ email: kim@cfa.harvard.edu
}

\begin{abstract}
Using Chandra and XMM-Newton X-ray observations of young, post-merger elliptical galaxies, we present X-ray characteristics of age-related observational results, by comparing with typical old elliptical galaxies in terms of X-ray properties of their low-mass X-ray binaries (LMXBs) and hot interstellar matter (ISM).
\end{abstract}

Keywords. galaxies: elliptical and lenticular, cD, X-rays: galaxies, X-rays: ISM

Stellar age in an elliptical galaxy has been measured and there are now a handful of elliptical galaxies with intermediate stellar age $(<5$ Gyr) which could link between Antennae-like merging systems and typical old elliptical galaxies (e.g,. Thomas et al. 2005). We find that young elliptical galaxies host more luminous $\left(>5 \times 10^{38} \mathrm{erg} \mathrm{s}^{-1}\right)$ LMXBs which are often distributed in a non-uniform way, indicating a possible connection with recent mergers (Kim et al. 2009, in preparation). Young elliptical galaxies tend to have a small amount of hot gas, with Lx(gas) comparable to or smaller than $\operatorname{Lx}(\mathrm{LMXB})$. By comparison, old elliptical galaxies have a wide range of $\mathrm{Lx}$ (gas) and some of them (e.g., group or cluster dominant galaxies) have Lx(gas) 10 or 100 times higher than $\operatorname{Lx}(\mathrm{LMXB})$.

One of the key observables to address the age effect is the abundance ratio of $F e$ to $\alpha$ elements, because of different production yields of SNe Ia and II. In the merger scenario of young elliptical galaxies, we would expect an enrichment of $\alpha$-elements from SN II, associated with the recent star formation. Based on the SN yields, [Si/Fe] would be close to 0.5 if $\mathrm{SNe}$ Ia are dominating, while the ratio would be close to 2.5 if SNe II are dominating. Typical old giant elliptical galaxies have [Si/Fe] close to solar (e.g., Kim \& Fabbiano 2004), indicating that the ejecta from both SNe II (from the early star formation) and SNe Ia (continuously added later) are well mixed. With added contribution from SNe II in young elliptical galaxies, we expect $[\mathrm{Si} / \mathrm{Fe}]$ to be between 1 and 2.5 solar. By carefully selecting young and old samples (e.g, similar $\sigma^{*}$ to avoid different $[\alpha / F e]^{*}$; weak AGN; deep Chandra observations to effectively remove LMXBs) and by rigorously analyzing the data (extracting X-rays only from $\mathrm{r}<30$ " to avoid a background problem; do not arbitrarily tying different elements), we find that [Si/Fe] is close to 1.5-2 solar in young E's (NGC 720 and NGC 3923), while [Si/Fe] is very close to solar in old E's (e.g, N4472, N4649). Given the limited sample and related uncertainties, our result is consistent with the expectation.

Finally, we note that while for X-ray faint galaxies the absolute abundances of individual elements are uncertain and controversial, the abundance ratio is better constrained because of strong correlations between errors of different elements.

\section{References}

Kim, D.-W. \& Fabbiano, G. 2004, ApJ 613, 933

Thomas, D. Maraston, C., Bender, R., \& de Oliveira, C. M. 2005, ApJ 621, 673 